\title{
Effects of photophoresis on the evolution of transitional circumstellar disks
}

\author{
F. Herrmann and A. V. Krivov
}

\author{
Astrophysikalisches Institut, Friedrich-Schiller-Universität Jena, Schillergäßchen 2-3, 07745 Jena, Germany \\ e-mail: fabian@astro.uni-jena.de
}

Received 19 July 2007 / Accepted 1 October 2007

ABSTRACT

\begin{abstract}
Although known for almost a century, the photophoretic force has only recently been considered in astrophysical context for the first time. In our work, we have examined the effect of photophoresis, acting together with stellar gravity, radiation pressure, and gas drag, on the evolution of solids in transitional circumstellar disks. We have applied our calculations to four different systems: the disks of HR 4796A and HD 141569A, which are several Myr-old AB-type stars, and two hypothetical systems that correspond to the solar nebula after disk dispersal has progressed sufficiently for the disk to become optically thin. Our results suggest that solid objects migrate inward or outward, until they reach a certain size-dependent stability distance from the star. The larger the bodies, the closer to the star they tend to accumulate. Photophoresis increases the stability radii, moving objects to larger distances. What is more, photophoresis may cause formation of a belt of objects, but only in a certain range of sizes and only around low-luminosity stars. The effects of photophoresis are noticeable in the size range from several micrometers to several centimeters (for older transitional disks) or even several meters (for younger, more gaseous, ones). We argue that due to gas damping, rotation does not substantially inhibit photophoresis.
\end{abstract}

Key words. stars: planetary systems: formation - stars: planetary systems: protoplanetary disks - stars: circumstellar matter celestial mechanics - stars: individual: HR 4796A - stars: individual: HD 141569A.

\section{Introduction}

The standard (core accretion) scenario of planet formation implies continuous growth of solids in a protoplanetary disk around a young star (see, e.g. Safronov 1972; Wetherill 1980; Shu et al. 1987; Lissauer 1993; Strom \& Edwards 1993; Weidenschilling \& Cuzzi 1993; Papaloizou et al. 1999; Blum \& Wurm 2000; Wurm \& Blum 2006; Henning et al. 2006; Meyer et al. 2007). Micron-sized dust grows step by step to larger bodies, until asteroid-sized planetesimals are reached. At sizes well below $1 \mathrm{~km}$, gravitational interactions between the objects play a minor role and the process is largely determined by interactions of solids with the ambient gas in the disk. Gas causes sedimentation, mixing, radial drift (Weidenschilling 1977; Kley et al. 1993; Takeuchi \& Artymowicz 2001; Brauer et al. 2007) and other effects that all dictate the spatial, size, and velocity distributions of grains and therefore the conditions for their growth. As the disk evolves, both gas and dust are gradually removed from the systems. In this process, the gas-to-dust ratio reduces from $\sim 100$ in the initial protoplanetary disk stage, at the ages on the order of $1 \mathrm{Myr}$, to vanishing values of $\ll 1$ at the debris disk stage after 10 Myr (Lawson et al. 2004; Haisch et al. 2005; Hollenbach et al. 2005; Takeuchi et al. 2005; Jayawardhana et al. 2006; Balog et al. 2007; Currie et al. 2007).

Transition from gas- and dust-rich, optically-thick protoplanetary disks to nearly gas-free, optically-thin debris disks is currently in the focus of interest of both observational and theoretical effort (Wieneke \& Clayton 1983; Morfill 1983, 1988; Weidenschilling \& Cuzzi 1993; Strom et al. 1993; Zuckerman \& Becklin 1993; Simon \& Prato 1995; Meyer \& Beckwith 2000; Ardila et al. 2005; Calvet et al. 2005; Hueso \& Guillot 2005; Augereau 2006; Bouwman et al. 2006; Eisner et al. 2006).
Examples of transitional objects are TW Hya, HR 4796A and HD 141569A, while the well-known system $\beta$ Pic already enters into the realm of almost gas-free debris disks (Thébault \& Augereau 2005). Resolved transitional disks exhibit radial structure in the form of rings and gaps, often alternating, and there is a lot of debate whether this structure is caused by gravity of hidden planets (Augereau et al. 1999b; Wyatt et al. 1999; Telesco et al. 2000) or by interaction of solids with the ambient gas component (Klahr \& Lin 2000; Takeuchi \& Artymowicz 2001; Besla $\& \mathrm{Wu} 2007)$. The first possibility is supported by the fact that the amount of gas remaining in these systems is probably no longer sufficient to form Jupiter's gas envelope (Chen \& Kamp 2004) and so, the planet formation process must already be finished. The second hypothesis is substantiated by simulations based on the observational estimates of the gas contents. These show that gas drag, acting together with other forces - stellar gravity and radiation pressure - could result in segregation of different-sized solids and radial fractionation of dust.

In this paper, we study the motion of different-sized solids under the combined action of stellar gravity, radiation pressure, and gas drag, to which we add photophoresis, an additional radial force that acts on particles in a gas disk which are exposed to the radiation field of the central star. Photophoresis, one of the lesser known forces of physics, has been first described by Ehrenhaft (1917). The force is caused by the following process. If an object is embedded in a thin gas and is exposed to an anisotropic radiation field (i.e. of a star), a gas molecule that becomes accommodated by the object's surface and rejected again will depart from the illuminated - and therefore warmer - side on average with a greater velocity then from the dark, colder one. Net momentum is transferred to the object, accelerating it away from the light source (note that for very small particles, 
the force can also be attractive - see Tehranian et al. 2001). Although known for almost a hundred years and successfully used in technical applications, such as the construction of optical traps (Steinbach et al. 2004), it has only recently been analyzed in astrophysical context. The first attempt to examine its influence on planetary formation was made by Krauss \& Wurm (2005). In their subsequent paper (Wurm \& Krauss 2006), they investigated how photophoresis might affect the formation of chondrules and the survival of calcium/aluminium-rich inclusions. Finally, Krauss et al. (2006) analyzed the effects imposed by photophoresis on the inner rim of a dusty protoplanetary disk. They assumed the disk to stay optically thick at all times, thus allowing only its inner edge to be influenced by photophoresis. Using an exponential law to describe particle growth and the $\alpha 1+1 \mathrm{D}$ turbulent model (Shakura \& Sunyaev 1973) used in Alibert et al. (2005) and Papaloizou \& Terquem (1999) for the evolution of the gas disk, Krauss and Wurm computed the motion of the inner edge. This is identical to the motion of the smallest particles, since, as will be shown subsequently, for solar luminosity systems, the velocity of outward motion of an object increases with its size in a certain size range (compare Fig. 3). In essence, they showed that the dust disk's edge first moves outward, pushed by photophoresis. After a few million years of time, it shrinks towards the star again, because gas pressure declines as disk dispersal proceeds.

This paper focuses on transitional disks. In these systems, the conditions are quite favorable for photophoresis to be efficient. Transitional disks are already optically thin, so that the particles are exposed to the strong radiation field. On the other hand, they are still sufficiently gas-rich. We would like to check whether, and to which extent, photophoresis may affect the radial migration of solids.

We use a single-body dynamics approach to investigate the behavior of a two-dimensional swarm of particles, the sizes of which are distributed in an interval ranging from several micrometers to several meters. The size of an individual particle, though, is being kept constant. We develop a simple theory for the long-term behavior of a distribution of solids experiencing gravity, radiation pressure, gas drag and photophoresis. Methodically, our study is similar to Takeuchi \& Artymowicz (2001), whose computations we generalize to the presence of photophoresis. To solve the equation of motion, we employ a modification of the semianalytic scheme used by Weidenschilling (1977). The results are applied to circumstellar disks with different gas density around stars of different luminosity.

In Sect. 2, we discuss the astrophysical status and parameters of the systems chosen for this study, as well as the formulas used for gas density and temperature in the disks. Section 3 provides formulas for different perturbing forces, including the photophoretic one. Section 4 deals with the equation of motion. In Sect. 5, we analyze the properties of the particles' radial motion and derive a formula for the radii of stable orbits. We also estimate the size ranges in which photophoresis should be taken into account. In Sect. 6, we examine the problem of particle rotation in order to check whether it might nullify the effect of photophoresis. In Sect. 7, the results are summarized and ideas for future research are presented.

\section{The systems}

Following Takeuchi \& Artymowicz (2001), we choose two transitional disks of particular interest for our study: HR 4796A and HD 141569A. We use these two systems in our work as model environments for the forces we wish to explore, as their properties are quite well known and reasonably accurate models exist for the physical conditions (gas density and temperature) in their disks.

The A0-type star HR 4796A, located at a distance of $67.1_{-3.4}^{+3.5} \mathrm{pc}$ from the sun (Hipparcos data), is a member of the TW-Hydrae-Association (TWA in the following, see Kastner et al. 2001). According to current knowledge, this is the young star association closest to our sun (Zuckerman \& Song 2004). The age of TWA can be estimated to be approximately $10 \mathrm{Myr}$. Since it has used up its molecular cloud completely, the only gas left in in the association is bound in the circumstellar disks. The stellar parameters of HR 4796A are estimated to be $M_{\star}=$ $2.5 M_{\odot}, L_{\star}=21.0 L_{\odot}$ (Koerner et al. 1998; Jayawardhana et al. 1998; Telesco et al. 2000). IRAS discovered that it emits $0.5 \%$ of its entire radiative power in the infrared part of the spectrum, which points to a high dust density in its neighborhood - in fact, HR 4796A is the dust-richest star in the Bright Star Catalog. Stauffer et al. (1995) estimate its age to be $8 \pm 2$ Myr, which makes it slightly younger than $\beta$ Pic (12 Myr). The structure of the dust disk around HR 4796A is highly complicated. First resolved images in thermal infrared were obtained by Koerner et al. (1998) and Jayawardhana et al. (1998). Wahhaj et al. (2005) conducted elaborate studies of the disk's structure, using data from MIRLIN at the Keck II telescope as well as $350 \mu \mathrm{m}$ measurements from the Caltech Submillimeter Observatory and Hubble Space Telescope scattered light images. Their studies point to a disk composed of an inner, exozodiacal dust ring located at roughly $r=4$ AU from the star and a wide, twocomponent outer dust belt consisting of a broad ring of $\sim 7 \mu \mathrm{m}$ grains stretching from 45 to $125 \mathrm{AU}$, and a narrower structure between 66 and $80 \mathrm{AU}$ consisting of $\sim 50 \mu \mathrm{m}$ grains. While the exozodiacal dust may be the product of an asteroid-type belt, the outer belt can be explained most naturally by the assumption that the grains are emitted by an exo-Kuiper swarm of planetesimals. Since the smallest ones are diffused rapidly by radiation pressure, they form a broad belt, while the larger ones stay closer to their area of origin. Also, asymmetric structures have been reported (Telesco et al. 2000). They may be caused by secular perturbations of one or several planets or by the stellar companion HR 4796B. Most probably, the disk is perturbed by planetary bodies as well as by the $\mathrm{B}$ component. In order to define the mass and number of planets, the orbit of HR 4796B would have to be known with greater precision.

The second object, HD 141569A, has the Hipparcos distance of $99 \pm 10 \mathrm{pc}$. Its parameters are estimated as $M_{\star}=$ $2.3 M_{\odot}, L_{\star}=22.4 L_{\odot}$ (Jura et al. 1993, 1998; van den Ancker et al. 1998) It is underluminous for its spectral type B9.5 Ve, which is a common occurrence for young A-type stars, found also at HR 4796A, $\beta$ Pic and 49 Ceti (Jura et al. 1998; Lowrance et al. 2000). Being, like HR 4796A, part of a multiple system with an M2 and an M4 component, the lower-mass companions which have not yet reached the main sequence can be used to find an estimate for the system's age of $5 \pm 3$ Myr (Weinberger et al. 2000). As in the HR 4796A case, the disk itself has a complex morphology (Weinberger et al. 1999; Augereau et al. 1999a; Fisher et al. 2000; Mouillet et al. 2001), consisting of two dust belts with radii of 200 and $325 \mathrm{AU}$, the centers of which are shifted by 20-30 AU in the direction of the system's semiminor axis. Between them a dust-free gap can be found at 250 AU. Also, inside of 150 AU the disk's luminosity declines to the level of background noise, which points to a strong depletion of dust in the system's inner region. The outer ring shows a tightly-wrapped spiral structure, which, according to the 
Table 1. Parameters of gas disk models.

\begin{tabular}{llllrr}
\hline \hline System & Example & $\rho_{0}\left[10^{-10} \mathrm{~kg} \mathrm{~m}^{-3}\right]$ & Density exponent $q$ & Luminosity $L_{\star} / L_{\odot}$ & Mass $M_{\star} / M_{\odot}$ \\
\hline lGlL & "older" SN & 3.12 & -2.75 & 1.0 & 1.0 \\
hGlL & "younger" SN & 156 & -2.75 & 1.0 & 1.0 \\
lGhL & HR 4796A* & 3.12 & -2.75 & 21.0 & 2.5 \\
hGhL & HD 141569A* & 156 & -2.25 & 22.4 & 2.3 \\
\hline
\end{tabular}

* Sources: Takeuchi \& Artymowicz (2001), their Table 1.

numerical calculations of Augereau \& Papaloizou (2004), can be produced by the gravitative perturbation of HD 141569B and C. The dust gap at $250 \mathrm{AU}$ may be caused by a planet of approximately Jovian mass, but it is not yet clear whether gas giants can form within a few million years at a distance of several hundred AU from the star (Wyatt 2005).

Both disks - HR 4796A and HD 141569A - surround luminous, early-type stars. Since we wish to explore the dependence of the particle dynamics on the stellar luminosity as well, we add two more, hypothetical systems with gas densities equal to those of HR 4796A and HD 141569A, but around a star of solar luminosity. They can be viewed as representations of the solar nebula at different stages of dissipation (Hollenbach et al. 1994; Hollenbach et al. 2000).

For gas density and temperature in all four model systems, we use standard power-law approximations (Hayashi et al. 1985):

$\frac{T}{1 \mathrm{~K}}=278\left(\frac{L_{\star}}{L_{\odot}}\right)^{1 / 4}\left(\frac{r}{1 \mathrm{AU}}\right)^{-1 / 2}$

and

$\frac{\rho_{\mathrm{g}}}{1 \mathrm{~kg} \mathrm{~m}^{-3}}=\rho_{0}\left(\frac{r}{1 \mathrm{AU}}\right)^{q}$,

where $\rho_{0}$ and $q$ are constants that vary from one system to another. To keep the treatment simple, we assume the exponent $q$ to be constant throughout each disk and do not use a "breakoff function" to describe the density drop beyond a certain radius $r_{\text {out }}$ (Takeuchi \& Artymowicz 2001). As will be shown subsequently, the outer part of the radial profile does not really matter, because photophoresis affects only the inner parts of the disk.

Altogether, our model contains three basic parameters: the gas density $\rho_{0}$ at $r=1 \mathrm{AU}$, the gas density exponent $q$ which controls the size of the gas disk, and the stellar luminosity $L_{\star}$. Our four model systems essentially explore the photophoretic effect in the "parameter rectangle" luminosity - gas density:

- lLhG (low luminosity, high gas content, corresponding to the solar nebula at an earlier stage),

- $l L l G$ (low luminosity, low gas content, solar nebula at a later stage),

- $h L h G$ (high luminosity, high gas content, HD 141569A), and

- $h L l G$ (high luminosity, low gas content, HR 4796A).

The parameters of all four systems are listed in Table 1.

\section{Forces}

In circumstellar nebulae, solid bodies experience a number of perturbing forces in addition to gravity, causing them to move along non-Keplerian orbits. In a first approximation, we can assume that they describe circular orbits, the radii of which shrink or grow in the course of time, depending on the size of the body and its distance from the star. We now introduce the different forces, presenting an analytic expression for each.

\subsection{Photophoresis}

While Rohatschek (1996) found a semi-empirical formula for the photophoretic force at all gas pressures, Beresnev et al. (1993) derived an analytic expression for spherical, nonrotating objects with a homogenous surface using an elaborate theoretical approach starting from the molecular velocity distribution function (compare also Tehranian et al. 2001). The acceleration due to the photophoretic force they found is:

$$
\begin{aligned}
a_{\text {phot }}= & \frac{I J_{1}}{4 s \rho_{\text {bulk }}} \sqrt{\frac{\pi \mu_{\mathrm{g}} m_{\mathrm{H}}}{2 k T}} \\
& \times \frac{\alpha_{\mathrm{E}} \Psi_{1}}{\alpha_{\mathrm{E}}+15 \Lambda K n\left(1-\alpha_{\mathrm{E}}\right) / 4+\alpha_{\mathrm{E}} \Lambda \Psi_{2}} .
\end{aligned}
$$

Here, $s$ is the particle radius, $I$ the radiation intensity, $J_{1}$ the asymmetry parameter that describes the accommodation of gas molecules to the particle's surface and light absorption. Assuming complete absorption and an accommodation probability of $100 \%$, we set $J_{1}=0.5$. The mean molecular weight of a gas of solar composition is denoted by $\mu_{\mathrm{g}}=2.34$ and the mass of a hydrogen atom by $m_{\mathrm{H}}$. Further, $k$ is Boltzmann's constant, $T$ the gas temperature and $\rho_{\text {bulk }}$ the density of the solid material. For icy aggregates, $\rho_{\text {bulk }} \approx 1000 \mathrm{~kg} \mathrm{~m}^{-3}$ is a good approximation. The energy accommodation coefficient $\alpha_{\mathrm{E}}$ is the fraction of molecules in contact with the surface that accommodate to the local temperature, which enables them to contribute to photophoresis. In our work we assume complete accommodation, and thus set $\alpha_{\mathrm{E}}=1$. The heat exchange parameter $\Lambda$, which describes the particle's thermal relaxation properties, is determined as

$\Lambda=\frac{k_{\mathrm{th}}+4 \epsilon \sigma T^{3} s}{k_{\mathrm{gas}}}$,

where $k_{\mathrm{th}}$ is the material's thermal conductivity, $\epsilon$ its emissivity, $\sigma$ the Stefan-Boltzmann constant and $k_{\text {gas }}$ the thermal conductivity of the gas. Assuming black bodies, we set $\epsilon=1$. The quantities $\Psi_{1}$ and $\Psi_{2}$ are given by

$$
\begin{aligned}
& \Psi_{1}=\frac{K n}{K n+(5 \pi / 18)}\left(1+\frac{2 \pi^{1 / 2} K n}{5 K n^{2}+\pi^{1 / 2} K n+\pi / 4}\right), \\
& \Psi_{2}=\left(\frac{1}{2}+\frac{15}{4} K n\right)\left(1-\frac{1.21 \pi^{1 / 2} K n}{100 K n^{2}+\pi / 4}\right),
\end{aligned}
$$

and $K n$ is the Knudsen number. It is defined as $K n \equiv L / s$, where $L=1 /\left(\sqrt{(32)} \pi n_{\text {gas }} r_{\mathrm{g}}^{2}\right)$ is the molecule's mean free path, with $r_{\mathrm{g}} \approx 10^{-10} \mathrm{~m}$ being the radius of gas molecules and $n_{\text {gas }}$ their number density.

Expression Eq. (3) for the photophoretic acceleration is valid for all Knudsen numbers. In the case of high Knudsen numbers (mean free paths of molecules are large compared to particle sizes), Eq. (3) reduces to (see Krauss \& Wurm (2005), their Eq. (1), and Beresnev et al. (1993), their Eq. (26))

$a_{\mathrm{phot}}=\frac{I p J_{1}}{4 \rho_{\mathrm{bulk}}\left(\Upsilon_{\mathrm{hc}}+\Upsilon_{\mathrm{rad}}+\Upsilon_{\mathrm{gas}}\right)}$, 

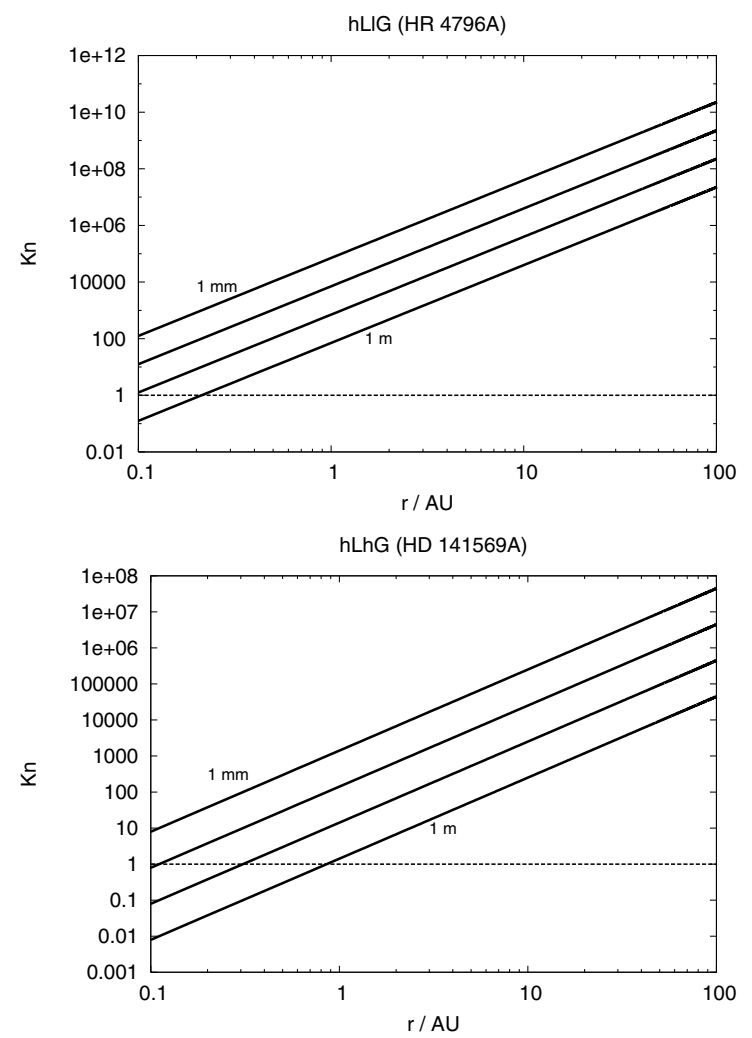

Fig. 1. Knudsen numbers $K n=L / s$ for systems with low (top) and high (bottom) gas content as a function of distance from the star. In each panel, four lines correspond to the particle radii of $1 \mathrm{~mm}, 1 \mathrm{~cm}, 10 \mathrm{~cm}$, and $1 \mathrm{~m}$. Since $L$ only depends on gas density, $K n$ does not vary with stellar luminosity.

where

$$
\begin{aligned}
\Upsilon_{\mathrm{hc}} & =k_{\mathrm{th}} T, \\
\Upsilon_{\mathrm{rad}} & =4 \sigma T^{4} \epsilon s, \\
\Upsilon_{\mathrm{gas}} & =p \sqrt{2 k T / \pi \mu_{\mathrm{g}} m_{\mathrm{H}}} s .
\end{aligned}
$$

Here, $p=n_{\text {gas }} k T$ is the gas pressure (assuming ideal gas). In transitional disks, we can usually assume to be in the high Knudsen number regime, except for large objects in the innermost parts of the disks (Fig. 1). The simplified expression (6) will allow us to find useful approximate solutions for the particles' stability radii (i.e. the radii of stable circular orbits). For large particles $(s \geq 10 \mathrm{~cm})$ and small distances from the star $(r \leq 1 \mathrm{AU})$, the more general expression (3) will be used in numerical calculations.

The terms $\Upsilon_{\text {hc }}, \Upsilon_{\text {rad }}$ and $\Upsilon_{\text {gas }}$ are related to three different processes which reduce the photophoretic effect. The first term, $\Upsilon_{\mathrm{hc}}$, corresponds to the transport of thermal energy through the object - i.e. heat conductivity, which reduces the temperature gradient, thus lowering the efficiency of photophoresis. The second one, $\Upsilon_{\text {rad }}$, describes thermal radiation from the particles' surface. Since it is proportional to $T^{4}$, much more energy is radiated from the warm than from the dark side, which results in a substantial reduction of the temperature gradient, and, therefore, of photophoresis. Obviously, this process gains efficiency with increasing temperature. Finally, the third term $\Upsilon_{\text {gas }}$ describes heat conduction away from the object's surface into the surrounding gas. Since it also shows a weak dependence on temperature $\left(\propto T^{1 / 2}\right)$, it causes a reduction of temperature gradient too.

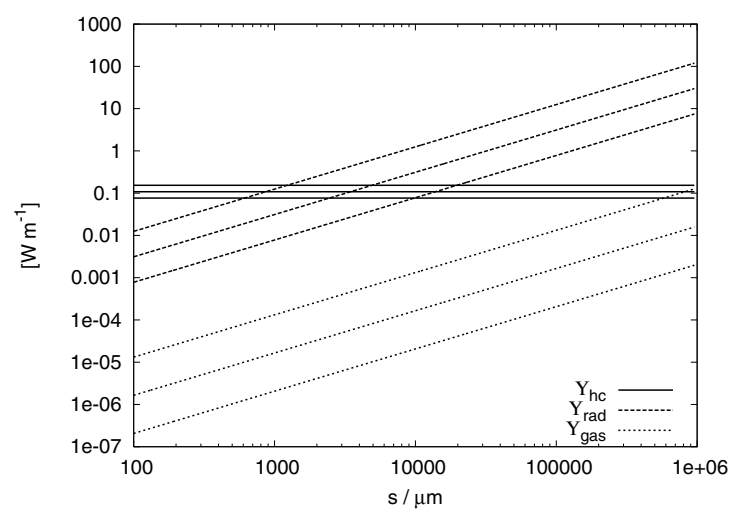

Fig. 2. Quantitative comparison of the three reduction processes for hLhG (HD 141569A) at $r=5,10,20 \mathrm{AU}$ (from above for each set of lines). For the other systems the result looks qualitatively similar, with $\Upsilon_{\text {gas }}$ being in the case of the gas-poorer systems completely negligible for all sizes $s$.

Krauss \& Wurm (2005) and Wurm \& Krauss (2006) use only the first term $\Upsilon_{\mathrm{hc}}$ in the denominator of Eq. (6), which is a good approximation for particles with $s \ll 1 \mathrm{~mm}$ and only in the case of low luminosity (i.e. solar-type) systems (see Sect. 5.2). In order to analyze the relative importance of the three "countereffects" $\Upsilon_{\text {hc }}, \Upsilon_{\text {rad }}$ and $\Upsilon_{\text {gas }}$, we plot them for HD 141569A as functions of particle size (Fig. 2) at three different distances.

Figure 2 shows that for fairly small particles $(s \leq 1 \mathrm{~mm})$, heat conduction $\left(\Upsilon_{\mathrm{hc}}\right)$ is the main reduction process. At larger sizes $(s \geq 10 \mathrm{~cm})$, radiation $\left(\Upsilon_{\text {rad }}\right)$ takes over. In the intermediate interval, both processes contribute to the reduction of photophoresis. At yet larger sizes $(s \geq 1 \mathrm{~m})$, heat conduction from the surface into the surrounding gas $\left(\Upsilon_{\text {gas }}\right)$ comes into play, approaching values similar to that of $\Upsilon_{\mathrm{hc}}$. As lower gas pressure dramatically reduces the importance of $\Upsilon_{\text {gas }}$, it is completely negligible for all object radii in the case of the gas-poor systems hLlG (HR 4796A) and ILlG.

\subsection{Radiation pressure}

Since radiation pressure is proportional to $r^{-2}$, it can be taken into account by introducing an "effective stellar mass" (Burns et al. 1979):

$M_{\mathrm{eff}}=M_{\star}(1-\beta)$,

where $\beta$ stands for the ratio of accelerations due to radiation pressure and gravity:

$$
\begin{aligned}
\beta & =\frac{a_{\mathrm{rad}}}{a_{\mathrm{grav}}}=\frac{0.5738 Q_{\mathrm{pr}}}{\rho_{\mathrm{bulk}}\left[\mathrm{g} \mathrm{cm}^{-3}\right] s[\mu \mathrm{m}]} \frac{L_{\star} / L_{\odot}}{M_{\star} / M_{\odot}} \\
& \equiv B\left(\frac{s}{1 \mu \mathrm{m}}\right)^{-1},
\end{aligned}
$$

with $Q_{\mathrm{pr}}$ being the radiation pressure efficiency. We will make use of the constant $B$ later. The effective "photogravitational" acceleration is then

$\boldsymbol{a}_{\mathrm{grav}, \mathrm{eff}}=-G \frac{M_{\mathrm{eff}}}{r^{3}} \boldsymbol{r}$

In our work, we simply set $Q_{\mathrm{pr}}=1$, assuming the solids to be black bodies. The reason is that generalization to non-black surfaces requires a change not only in the emissivity, but also in the asymmetry parameter $J_{1}$. The latter is not known for realistic 
materials, and its determination requires complicated numerical procedures (see, e.g. Mackowski 1989). Analytical solutions are possible, but only in the cases of high or low absorption coefficients (see Arnold \& Lewittes 1982).

\subsection{Gas drag force}

Unlike the other three forces (gravity, radiation pressure, photophoresis), gas drag is not a radial force. Since it results from the momentum transferred to the body by molecules impinging on it as it moves through the gas, the force vector is antiparallel to the relative velocity $\Delta v$ of the dust grain with respect to the gas.

To calculate gas drag, we first need a model for the motion of the gas component of the circumstellar nebula. Since the pressure gradient supports gas against stellar gravity, it travels on circular orbits with a sub-Keplerian speed

$v_{\mathrm{g}}=v_{\mathrm{K}} \sqrt{1-\eta}$,

and angular velocity

$\Omega_{\mathrm{g}}=\Omega_{\mathrm{K}} \sqrt{1-\eta}$,

where $v_{\mathrm{K}}=\sqrt{G M_{\star} / r}$ is the Keplerian circular velocity, $\Omega_{\mathrm{K}}=$ $v_{\mathrm{K}} / r=\sqrt{G M_{\star} / r^{3}}$ the corresponding angular velocity, and $\eta$ is the ratio of pressure gradient force to gravity:

$\eta=-\frac{1}{r \Omega_{\mathrm{K}}^{2} \rho_{\mathrm{g}}} \frac{\mathrm{d} p}{\mathrm{~d} r}$.

The $\eta$ ratio can be rewritten as:

$$
\begin{aligned}
\eta= & 1.1 \times 10^{-3}\left(\frac{1}{2}-q\right)\left(\frac{\mu_{\mathrm{g}}}{2.34}\right)^{-1}\left(\frac{L_{\star}}{L_{\odot}}\right)^{1 / 4} \\
& \times\left(\frac{M_{\star}}{M_{\odot}}\right)^{-1}\left(\frac{r}{1 \mathrm{AU}}\right)^{1 / 2} \equiv E\left(\frac{r}{1 \mathrm{AU}}\right)^{1 / 2} .
\end{aligned}
$$

If the motion of the particles is subsonic $\left(\Delta v \ll v_{\mathrm{T}} \approx c_{\mathrm{S}}\right.$ where $c_{\mathrm{S}}$ is the speed of sound - this is always the case in the transitional disks), the gas drag acceleration is given by (see Takeuchi \& Artymowicz 2001):

$\boldsymbol{a}_{\mathrm{D}}=-\frac{3 \rho_{\mathrm{g}}}{4 \rho_{\mathrm{bulk}} s} v_{T} \Delta \boldsymbol{v}$,

where

$v_{\mathrm{T}}=\frac{4}{3}\left(\frac{8 k T}{\pi \mu_{\mathrm{g}} m_{\mathrm{H}}}\right)^{\frac{1}{2}}=\frac{4}{3} \times\left\langle v_{\text {therm }}\right\rangle$

is $4 / 3$ times the mean thermal velocity.

The reaction of particles to the gas drag force critically depends on their size. Small objects adjust their velocity instantaneously, they are swept along with the gas component. Large ones react only sluggishly to gas drag, taking a longer time to change their velocity substantially. In order to create a quantitative measure for the tendency of solids to be influenced by gas drag, the stopping time is introduced. We denote the time needed for a particle injected into the gas to be slowed down to $e^{-1}$ times its initial velocity by $t_{\mathrm{s}}=\Delta v / a_{\mathrm{D}}$. The dimensionless stopping parameter $T_{\mathrm{s}}$ is defined by:

$T_{\mathrm{s}} \equiv t_{\mathrm{s}} \Omega_{\mathrm{K}} \approx \frac{4 \rho_{\text {bulk }} s v_{\mathrm{K}}}{3 \rho_{\mathrm{g}} r v_{\mathrm{T}}}$.

The right-hand side approximation holds in the case of subsonic motion. It renders the stopping time independent of the particle's momentary velocity.

\section{Equation of motion}

The equation of motion of a dust particle is:

$\frac{\mathrm{d}^{2} \boldsymbol{r}}{\mathrm{d} t^{2}}=\boldsymbol{a}_{\text {grav }, \text { eff }}+\boldsymbol{a}_{\mathrm{phot}}+\boldsymbol{a}_{\mathrm{D}}$

Being interested in the radial motion of the bodies, we now consider the radial component of the equation of motion (20). In the reference frame corotating with the gas, it takes the form

$\frac{\mathrm{d}^{2} r}{\mathrm{~d} t^{2}}-\Omega_{\mathrm{g}}^{2} r=-a_{\text {grav }, \text { eff }}+a_{\text {phot }}$

The acceleration $\mathrm{d}^{2} r / \mathrm{d} t^{2} \equiv \Delta g$ of the particle in the corotating frame can be interpreted as the "residual gravity". It computes to

$\Delta g=-a_{\text {grav, eff }}+a_{\text {phot }}+\Omega_{\mathrm{g}}^{2} r=(\beta+\chi-\eta) \Omega_{\mathrm{K}}^{2} r$,

where $\chi \equiv a_{\text {phot }} / a_{\text {grav }}$ is the photophoresis-to-gravity ratio. Particles experience the inward-directed residual acceleration $\eta \Omega_{\mathrm{K}}^{2} r$ and the outward-directed accelerations due to photophoresis $\left(\chi \Omega_{\mathrm{K}}^{2} r\right)$ and radiation pressure $\left(\beta \Omega_{\mathrm{K}}^{2} r\right)$.

Assuming now that the solid particle moves at a Keplerian circular speed, we can write

$\frac{v_{\mathrm{g}}^{2}}{r}=\frac{v_{\mathrm{K}}^{2}}{r}+\Delta g$.

The relative velocity is then approximately

$\Delta v=v_{\mathrm{K}}-v_{\mathrm{g}} \approx-\left(\frac{\Delta g}{2 a_{\text {grav }}}\right) v_{\mathrm{K}}$.

We now use $\Delta g$ to derive an approximation for the radial drift velocity of the particles. We can discern two limiting cases:

- Small particles are swept along with the gas. They stay on circular orbits, while moving with sub-Keplerian velocity, which causes them to experience the residual gravity pull $\Delta g$.

- Large particles are decoupled from gas. They move on Keplerian orbits, experiencing a head wind that gradually reduces their angular momentum.

In the first case, the radial drift velocity $v_{\mathrm{r}} \equiv \mathrm{d} r / \mathrm{d} t$ computes to (see Weidenschilling 1977, Sects. 4.1, 4.2):

$v_{\mathrm{r}, \text { small }}=t_{\mathrm{s}} \Delta g=T_{\mathrm{s}}(\beta+\chi-\eta) r \Omega_{\mathrm{K}}$.

In the second case, the orbit decays at a rate

$v_{\mathrm{r}, \text { large }}=-\frac{r}{t_{\mathrm{s}}} \frac{2}{v_{\mathrm{K}}} \Delta v=\frac{r}{t_{\mathrm{s}}} \frac{\Delta g}{a_{\text {grav }}}=\frac{(\beta+\chi-\eta) r \Omega_{\mathrm{K}}}{T_{\mathrm{s}}}$.

These two formulas can be combined into (see Takeuchi \& Artymowicz 2001, Sect. 3.3):

$v_{\mathrm{r}}=\frac{\beta+\chi-\eta}{T_{\mathrm{s}}+T_{\mathrm{s}}^{-1}} v_{K}$

In Fig. 3, we plot the absolute value of $v_{\mathrm{r}}$ over $s$ for $r=10 \mathrm{AU}$, both with and without photophoresis. The points where $v_{\mathrm{r}}=0$ are shifted to larger sizes by photophoresis. Note that for larger sizes, radial velocities depend almost exclusively on gas density since for them gas drag ( $\eta$ in Eq. (27)) becomes the main driving force of radial migration.

In what follows, differential Eq. (27) for $r(t)$ will be studied analytically and solved numerically. In the realm of large radial velocities (or equivalently, small particles), $r(t)$ should be computed from the "exact" equation of motion (20). However, our numerical tests have shown Eq. (27) to be accurate enough for all particle sizes larger than $\sim 100 \mu \mathrm{m}$. 

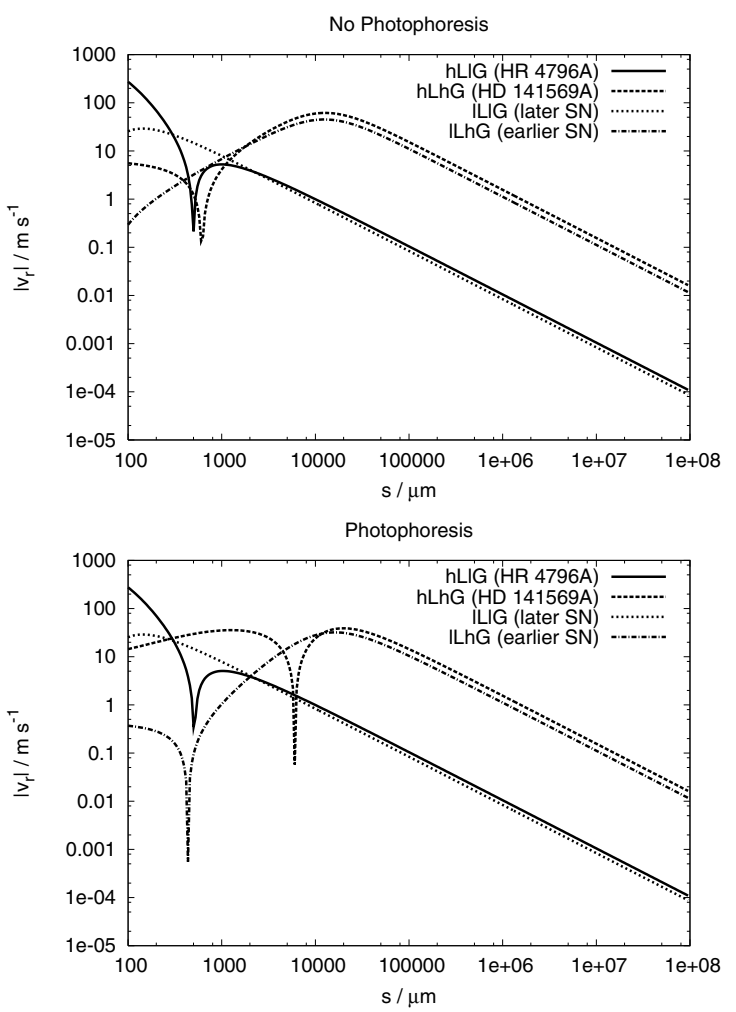

Fig. 3. The absolute values of the radial velocities at $r=10 \mathrm{AU}$. The minima correspond to the sizes for which $v_{\mathrm{r}}=0$.

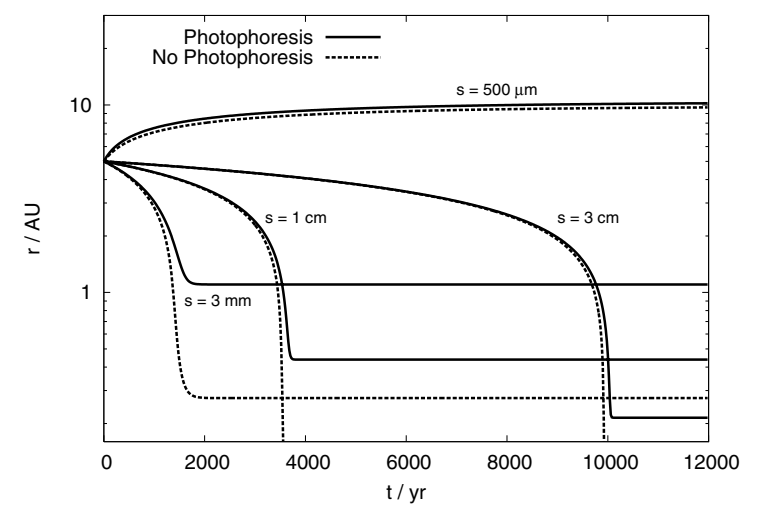

Fig. 4. Distance as a function of time for particles of four different radii in the hLlG system (HR 4796A). Dashed lines: without photophoresis, solid: with photophoresis. The lower border of the plot corresponds to the evaporation limit $r_{\text {evap }}=0.16 \mathrm{AU}$ (defined by black-body equilibrium temperature $T=1500 \mathrm{~K}$ ).

\section{Results}

\subsection{Radial motion}

We used Eq. (27) to calculate the radial drift of different-sized particles. What happens, in short, is that the particles migrate inward or outward, until they reach stable circular orbits on which gravity, centripetal force (in the particle's own inertial system), photophoresis and radiation pressure balance each other, thus permitting circular motion $\left(v_{\mathrm{r}}=0\right)$, while at the same time $\Delta v=0$, i.e. the particle travels at the same speed as the gas.

As an example, Fig. 4 shows the $r(t)$-curves for particles of four different sizes with and without photophoresis in the system hLlG (HR 4796A). The particles start on circular orbits with $r_{0}=5 \mathrm{AU}$. In fact, initial circularity is not important, because elliptic orbits are quickly circularized by the gas drag force. The initial value $r_{0}$ does not matter either, because the particles migrate towards their equilibrium orbits within several thousand years. While small, outward migrating bodies travel slowly towards their equilibrium distance $r_{\text {stab }}(s)$, approaching it asymptotically, the larger, inward migrating ones almost "drop" onto their stability orbits, being stopped almost instantaneously. Note, however, that "instantaneous" here refers to a deceleration process taking several centuries. Therefore, the large particles travel essentially on Keplerian orbits, corresponding to the second case described in Sect. 4. With increasing particle size, though, this process takes longer, as larger objects experience weaker drag acceleration than smaller ones. Another conclusion from Fig. 4 is that the stability radii are pushed outward by photophoresis.

\subsection{Equilibrium distance}

We now calculate the equilibrium distance which, according to Eq. (27), must satisfy

$\beta+\chi-\eta=0$.

From this, an implicit expression for the radius $r_{\text {stab }}(s)$ of the stable orbit can be derived:

$$
r_{\text {stab }}(s)=\frac{v_{K}^{2}\left(r_{\text {stab }}\right)\left(1-\eta\left(r_{\text {stab }}\right)\right)}{a_{\text {grav, eff }}\left(r_{\text {stab }}, s\right)-a_{\text {phot }}\left(r_{\text {stab }}, s\right)} .
$$

The equilibrium distance $r_{\text {stab }}(s)$ depends on the system's parameters as well as on the particle size. Figure 5 shows solutions of Eq. (29), computed with and without photophoresis. It can be clearly seen that photophoresis significantly increases the radii of stable orbits - as expected: photophoresis pushes particles away from the star. The curves exhibit a characteristic shape: after branching off from the solutions computed without photophoresis, they flatten. While for hLhG there is only a small region in which the curve is flatter, the two low-luminosity systems have well-developed plateaus, the length and distance from the star of which increase with gas density. The reason for this is explained below. Note also, that the plateaus in the $r_{\text {stab }}(s)$-curve correspond to areas, where solids have a higher surface density. At a certain particle size, the curves drop to zero suddenly. This is a consequence of mean free path becoming smaller than particle sizes - in this area, Eq. (6) is not a good approximation anymore, and Eq. (3) should be used instead to calculate the photophoretic acceleration. Because in the low Knudsen number regime photophoresis decreases in strength as body size increases, Eqs. (28) and (29) do not have solutions above a certain body radius, which causes $r_{\text {stab }}(s)$ to drop to zero, so that there are no stable orbits for solids above this critical size. These considerations are visualized in Fig. 6, where $\eta$ and $\chi$ are plotted as functions of $r$ for different radii $s$. The third force parameter, $\beta$, can be neglected for large particles ( $s \gg 100 \mu \mathrm{m})$, and therefore is not shown. We can identify two cases. For small bodies, the $\chi(r)$-curve has two intersections with $\eta(r)$. This means that in a certain size range, (28)-(29) have got two solutions. In our work we consider only the one farther out from the star, since in transitional disks we expect most particles to drift inward from larger radii, where they are produced by collisions between left-over planetesimals in exo-Kuiper belts. Besides, for three out of four systems (hGlL is the exception), the inner solution lies in the sublimation zone. As $s$ increases further, the $\chi(r)$-curve moves downward until it no longer crosses the $\eta$-curve, and thus no solution of (28)-(29) remains. This means that above a certain size no stable orbits exist. 

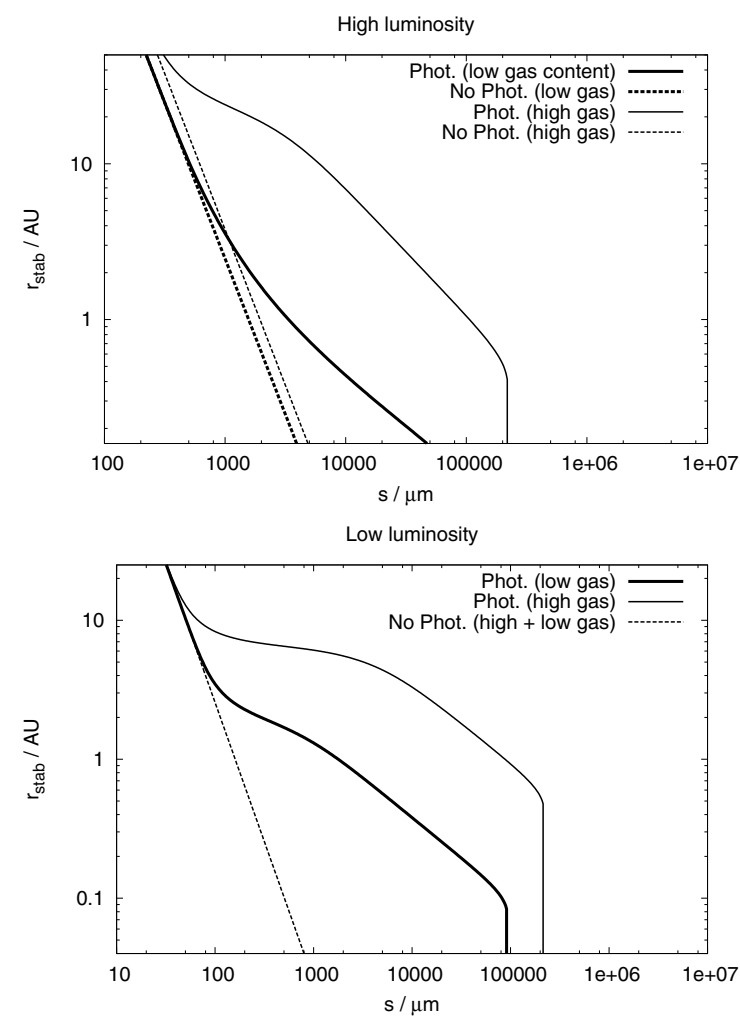

Fig. 5. Stability radii as functions of particle radius $s$ for two highluminosity systems (top) and two low-luminosity ones (bottom). In the bottom panel, the "No Photophoresis" curve is identical for both systems, as they differ only in the value of gas density, which does not affect the stability condition if photophoresis is not taken into account ( $\eta$ only depends on the density exponent $q$, not its absolute value - see Eq. (16)). Again, the lower borders of the plots correspond to the evaporation limits.

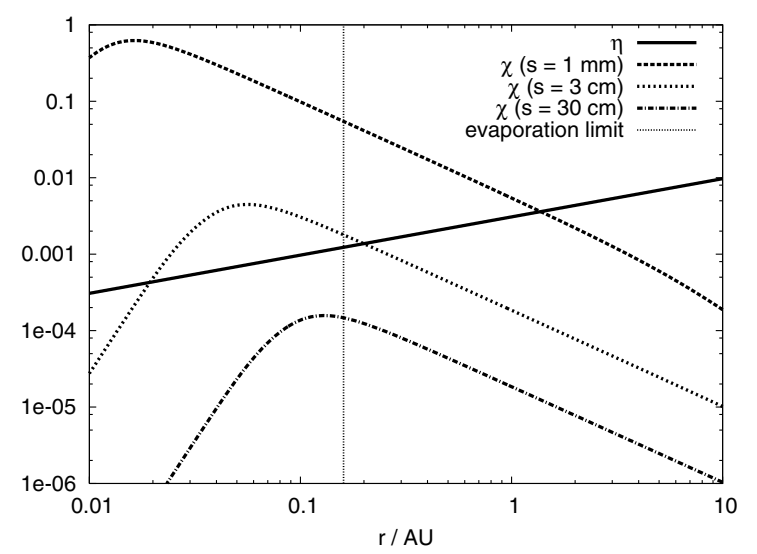

Fig. 6. The force ratios $\eta$ and $\chi$ as functions of $r$ for different particle sizes, in the hLlG system (HR 4796A). The number of intersections between the curves corresponds to the number of solutions of (28)-(29).

Next, we determine how the exact shape of $r_{\text {stab }}(s)$ is influenced by the choice of system parameters. Therefore, in Fig. 7, we plot the stability radii for different choices of $L_{\star}, \rho_{0}$ and $q$. Note that, when varying $L_{\star}$ in the bottom panel of Fig. 7, we change $M_{\star}$ accordingly. Analyzing the plots in Fig. 7, we can identify the following dependencies:

- Higher gas density $\rho_{0}$ moves the curves' middle parts to larger $r$, which is, of course, a consequence of photophoresis being directly dependent on gas pressure. Also, the limiting particle size beyond which no stable orbits exist depends almost exclusively on $\rho_{0}$, while the influence of the other parameters is rather weak.

- The density exponent $q$, which determines the slope of $\rho(r)$, also controls the slope of $r_{\text {stab }}(s)$ : a lower absolute value of $q$ produces a steeper curve, as the gas disk spreads to a greater distance from the star.

- Stellar luminosity $L_{\star}$ determines the characteristic shape of the $r_{\text {stab }}(s)$ curve. While large luminosities produce a monotonically decreasing curve, lower (i.e. solar) ones generate a plateau at a certain distance from the star, which gives rise to a concentration of particles of different sizes in that region.

The mechanism behind the third effect is to be found in the denominator of Eq. (6). As we have seen in Fig. 2, in different size ranges three different processes reduce the photophoretic effect - heat conduction in small particles, radiation for larger ones and heat conduction into the surrounding gas for very large objects. If the stellar luminosity is low, radiation becomes important only for relatively large sizes, while for smaller objects only $\Upsilon_{\mathrm{hc}}$ is relevant in Eq. (6). As $\Upsilon_{\mathrm{hc}}$ is independent of size, the resulting curve tends to run parallel to the $s$-axis, creating the plateau.

In the following, we formulate these considerations quantitatively. We are going to compute solutions of Eq. (29) for very large and very small particles, using the simplified largeKnudsen approximation for the photophoretic acceleration given in Eq. (6). We need to calculate the following ratios:

$\chi\left(\Upsilon_{\mathrm{hc}}\right)=\frac{a_{\mathrm{phot},} \Upsilon_{\mathrm{hc}}}{a_{\text {grav }}}$

and

$\chi\left(\Upsilon_{\mathrm{rad}}\right)=\frac{a_{\mathrm{phot},} \Upsilon_{\mathrm{rad}}}{a_{\text {grav }}}$,

where $a_{\text {phot, }} \Upsilon_{\mathrm{hc}}$ and $a_{\mathrm{phot}}, \Upsilon_{\mathrm{rad}}$ denote the accelerations due to (large Knudsen regime) photophoretic force using exclusively $\Upsilon_{\text {hc }}$ and $\Upsilon_{\text {rad }}$ in the denominator, respectively. The first case is a good approximation for small particle radii $(s \leq 1 \mathrm{~mm}$, compare Fig. 2), the second one for larger, but sub-meter objects $(1 \mathrm{~mm} \leq s \leq 1 \mathrm{~m})$.

The quantities $\chi\left(\Upsilon_{\mathrm{hc}}\right)$ and $\chi\left(\Upsilon_{\mathrm{rad}}\right)$ can be computed from the following expressions:

$$
\begin{aligned}
\chi\left(\Upsilon_{\mathrm{hc}}\right)= & 1.0 \times 10^{8}\left(\frac{M_{\star}}{M_{\odot}}\right)^{-1}\left(\frac{L_{\star}}{L_{\odot}}\right)\left(\frac{\mu_{\mathrm{g}}}{2.34}\right)^{-1} \\
& \times\left(\frac{\rho_{\mathrm{d}}}{1000 \mathrm{~kg} \mathrm{~m}^{-3}}\right)^{-1} \rho_{0}\left(\frac{r}{1 \mathrm{AU}}\right)^{q} \\
\equiv & Z\left(\Upsilon_{\mathrm{hc}}\right)\left(\frac{r}{1 \mathrm{AU}}\right)^{q}
\end{aligned}
$$

and

$$
\begin{aligned}
\chi\left(\Upsilon_{\mathrm{rad}}\right)= & 2.1 \times 10^{10}\left(\frac{M_{\star}}{M_{\odot}}\right)^{-1}\left(\frac{L_{\star}}{L_{\odot}}\right)^{1 / 4}\left(\frac{\mu_{\mathrm{g}}}{2.34}\right)^{-1} \\
& \times\left(\frac{\rho_{\mathrm{d}}}{1000 \mathrm{~kg} \mathrm{~m}^{-3}}\right)^{-1} \rho_{0}\left(\frac{r}{1 \mathrm{AU}}\right)^{q+3 / 2}\left(\frac{s}{1 \mu \mathrm{m}}\right)^{-1} \\
\equiv & Z\left(\Upsilon_{\mathrm{rad}}\right)\left(\frac{r}{1 \mathrm{AU}}\right)^{q+3 / 2}\left(\frac{s}{1 \mu \mathrm{m}}\right)^{-1} .
\end{aligned}
$$

It is easy to show that in the first (small-particle) case, Eq. (29) can be solved for $s$ :

$$
\frac{s_{\text {stab, small }}(r)}{1 \mu \mathrm{m}}=\frac{B}{E(r[\mathrm{AU}])^{1 / 2}-Z\left(\Upsilon_{\mathrm{hc}}\right)(r[\mathrm{AU}])^{q}} .
$$



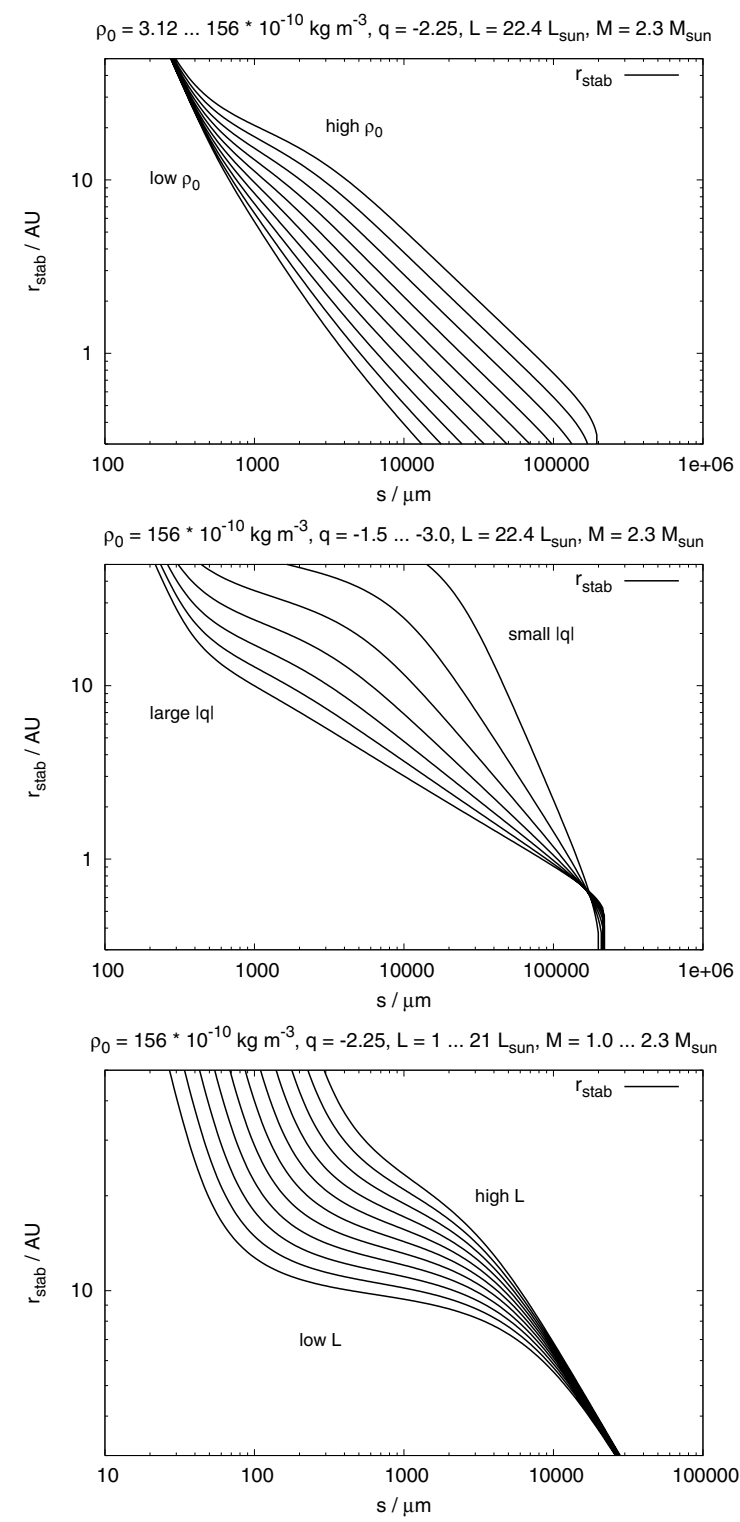

Fig. 7. Dependence of the equilibrium distance $r_{\text {stab }}(s)$ on the gas density $\rho_{0}$, the density exponent $q$, and stellar luminosity $L_{\star}$. Top: $\rho_{0}$ running, $q$ and $L_{\star}$ fixed. Middle: $q$ running, $\rho_{0}$ and $L_{\star}$ fixed. Bottom: $L_{\star}$ running, $q$ and $\rho_{0}$ fixed. The parameter values are indicated on top of each panel.

For definition of $B$ and $E$, see Eqs. (11) and (16). This allows us to calculate the size of particles on stable orbits at a certain stellar distance $r$. Since $q$ is negative, $s_{\text {stab, small }}$ tends to infinity as $r$ approaches zero. This produces the curve's plateau, i.e. the concentration belt. From Eq. (34), the belt's radius computes to:

$r_{\text {belt }}=\left(\frac{E}{Z\left(\Upsilon_{\mathrm{hc}}\right)}\right)^{1 /(q-1 / 2)}$.

For the four systems considered, the resulting distances are listed in Table 2.

In the second (large-particle) case, the corresponding expression

$\frac{s_{\text {stab }, \text { large }}(r)}{1 \mu \mathrm{m}}=\frac{B(r[\mathrm{AU}])^{-1 / 2}+Z\left(\Upsilon_{\mathrm{rad}}\right)(r[\mathrm{AU}])^{q+1}}{E}$

can be derived.
Table 2. Existence and parameters of the belt.

\begin{tabular}{lrl}
\hline \hline System & $r_{\text {belt }} /$ AU & $C$ \\
\hline lL1G ("older" SN) & 2.0 & 0.8 \\
lLhG ("younger" SN) & $\mathbf{6 . 5}$ & $\mathbf{2 . 6}$ \\
hL1G (HR 4696A) & 4.0 & 0.1 \\
hLhG (HD 141569A) & 22.8 & 0.07 \\
\hline
\end{tabular}

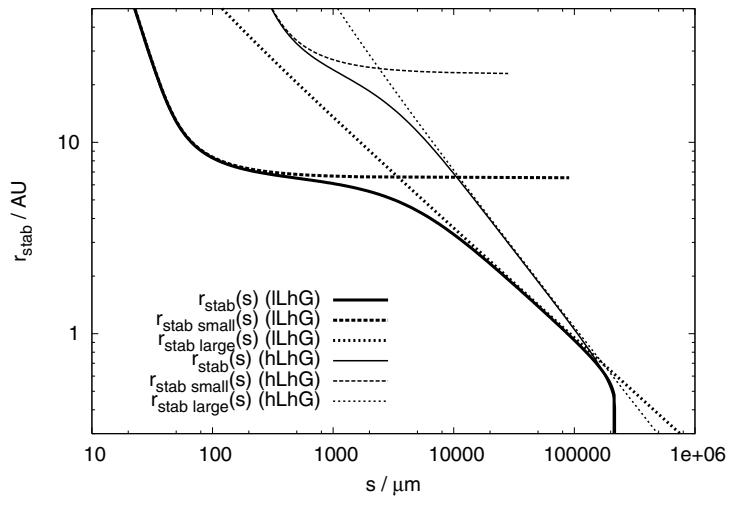

Fig. 8. "Exact" function $r_{\text {stab }}$ (solid lines) and its two approximations (dashed lines: Eq. (34); dotted: Eq. (36)), for the ILhG (thick lines) and the hLhG (thin) systems. Note how the behavior of $r_{\text {stab, small }}$ produces the curve's plateau - in the higher luminosity case, $r_{\text {stab, large "takes }}$ over" at smaller particle sizes, thus keeping $r_{\text {stab, small }}$ from producing the effect.

The condition for the formation of the belt is that at its position, the value of $s_{\text {stab, large }}$ has to be sufficiently larger

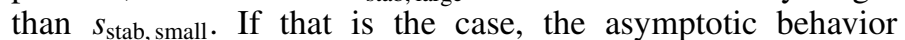
of $s_{\text {stab, small }}$ is seen in the "exact" curve too, otherwise it is overridden by $s_{\text {stab, large }}$ (see Fig. 8). To evaluate this condition numerically, we set

$r_{\text {belt }}^{\prime}=r_{\text {belt }}+\Delta r$

where for $\Delta r$ a sufficiently small value has to be chosen. We use $\Delta r=0.1 \mathrm{AU}$. This is necessary because $s_{\mathrm{stab} \text {, small }}$ cannot be computed at $r_{\text {belt }}$, as the denominator of Eq. (34) becomes zero at this point. Then, the ratio of the two approximating functions at $r_{\text {belt }}^{\prime}$ can be used as a measure of how pronounced the belt is:

$C \equiv s_{\text {stab, large }}\left(r_{\text {belt }}^{\prime}\right) / s_{\text {stab, small }}\left(r_{\text {belt }}^{\prime}\right)$.

The values of $C$ are listed in Table 2. Comparing with Fig. 5, we find that for $C \ll 1$, the plateau does not appear at all, or is only marginal. For $C \approx 1$, the belt is well developed. The particle size range it encompasses and its degree of concentration (i.e. the "flatness" of the curve in that area) increase with $C$, which makes it a direct measure for the system's tendency to produce a belt. In fact, other choices for $\Delta r$ are possible - for them, the values with which $C$ has to be compared, have to be changed. For $\Delta r=0.1 \mathrm{AU}$, the critical value above which well-formed belts appear is $C=1$.

We see that low luminosities and high gas densities are crucial for belt formation. Since $\Upsilon_{\text {rad }} \propto T^{4} \propto L_{\star}$, with rising luminosity $\Upsilon_{\text {rad }}$ becomes important at smaller particle sizes, superseding $\Upsilon_{\mathrm{hc}}$ before the plateau of $s_{\mathrm{stab} \text {, small }}$ is reached. Because they increase the overall strength of photophoresis, higher gas densities push the plateau outward, reducing its slope - and thus increasing the degree of particle concentration in the belt.

Figure 8 demonstrates how the exact solution of Eq. (29) is approximated by Eqs. (34) and (36) in different particle size ranges for the $1 \mathrm{LhG}$ and hLhG models. 


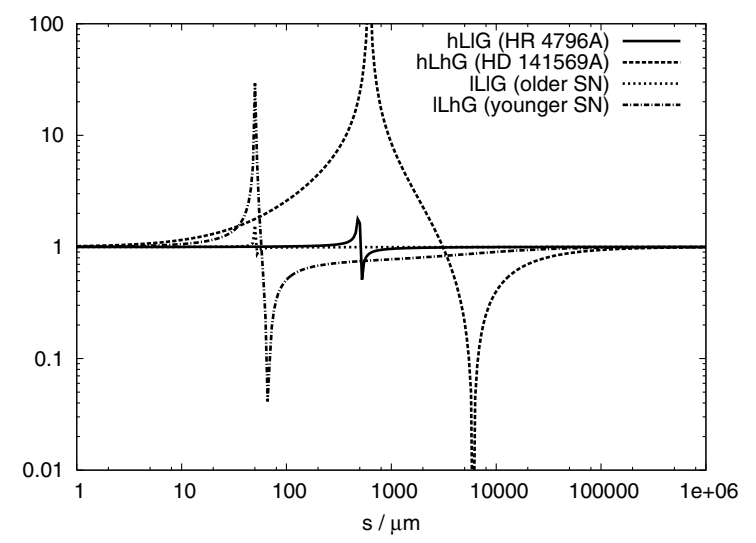

Fig. 9. Ratio of radial velocities, computed with and without photophoresis. The stronger the deviation from unity, the more important is photophoresis. The stellar distance is $r=10 \mathrm{AU}$.

Table 3. Size range of particles affected by photophoresis.

\begin{tabular}{lrl}
\hline \hline System & $s_{\min }$ & $s_{\max }$ \\
\hline lLlG ("older" SN) & $9 \mu \mathrm{m}$ & $3 \mathrm{~cm}$ \\
lLhG ("younger" SN) & $1 \mu \mathrm{m}$ & $1.5 \mathrm{~m}$ \\
hLlG (HR 4696A) & $10 \mu \mathrm{m}$ & $3.2 \mathrm{~cm}$ \\
hLhG (HD 141569A) & $1 \mu \mathrm{m}$ & $6 \mathrm{~m}$ \\
\hline
\end{tabular}

\subsection{Particle size range}

As we have seen, particles of different sizes are influenced by photophoresis to a different extent. Its relative importance depends on their particle radius, declining for very small (micrometer-range) and very large (above meter-range) objects. In between, photophoresis plays an important role. In order to analyze the critical size range, we plot the ratio $x \equiv\left|v_{\mathrm{r} \text {, phot }} / v_{\mathrm{r} \text {, no phot }}\right|$ as a function of $s$ in Fig. 9 , where $v_{\mathrm{r} \text {, phot }}$ and $v_{\mathrm{r} \text {, no phot }}$ are the radial velocity with and without photophoresis respectively. The distance is set to $r=10 \mathrm{AU}$. Note that the interval between the two peaks corresponds to the size range in which the two $v_{\mathrm{r}}$ have got different signs, because the stability radius with photophoresis is greater than $10 \mathrm{AU}$, while the one calculated without photophoresis is smaller.

If we use the criterion that $|x-1| \geq 0.001$, photophoresis has to be taken into account in the size ranges listed in Table 3. Note, however, that for $s \leq 10 \mu \mathrm{m}$, the above evaluation is no longer necessarily valid, as for small particles, the photophoretic force can reverse its direction (Tehranian et al. 2001). Also, for hLhG (HD 141569A), $\beta\left(s_{\min }\right)$ is greater than 0.5 , thus these particles are $\beta$-meteoroids which escape from the system.

\section{Rotation of particles}

Until now, we considered only nonrotating particles. We have to check whether this assumption is realistic, since the rotation of bodies can transport thermal energy from the dark to the lit side, thus banishing the photophoretic effect. There is a number of different mechanisms that can induce particle rotation:

- Collisionally induced rotation. During the formation of bodies as well as during their life, they collide with other particles, which can change their orbits as well as transfer angular momentum to them, spinning them up.

- Rotation induced by gas drag or radiation forces. If particles are not exactly spherical, any force acting on their surface will change their angular momentum. For instance, radiation pressure may spin-up the particles (the so-called windmill effect, Paddack \& Rhee 1975), but may also stabilize/align then (Draine \& Weingartner 1996). Such effects are beyond the scope of this paper.

Whether rotation is able to subdue photophoresis depends on four different timescales: $t_{\text {coll }}$, the typical time between two collisions; $t_{\mathrm{s}}$, the gas coupling time which also determines the time needed to slow down rotation; $t_{\text {heat }}$, the thermal relaxation time needed to establish a stable heat gradient within the particle; and $t_{\text {rot }}$, the typical rotation period.

The collisional time $t_{\text {coll }}$ can be estimated in a standard way:

$t_{\text {coll }}=\frac{1}{n_{\mathrm{p}} v \sigma_{\text {coll }}}$,

where $n_{\mathrm{p}}$ is the particle number density, $v$ the collisional velocity and $\sigma_{\text {coll }}=\pi\left(s_{1}+s_{2}\right)^{2}$ is the collisional cross section for spherical particles of radii $s_{1}$ and $s_{2}$. With

$n_{\mathrm{p}}=\frac{\rho_{\mathrm{d}}}{\rho_{\text {bulk }} \frac{4}{3} \pi s^{3}}$,

and the standard assumption $\rho_{\mathrm{d}} / \rho_{\mathrm{g}}=10^{-2}$, we get for particles of equal size (cf. Krauss et al. 2006, their Eq. (15)):

$t_{\text {coll }}=33 \frac{\rho_{\text {bulk }} s}{\rho_{\mathrm{g}} v}$.

The stopping time $t_{\mathrm{s}}$ was computed in Sect. 3.3, Eq. (19). Finally, the thermal relaxation time $t_{\text {heat }}$ is given by (see Krauss \& Wurm 2005, Eq. (6)):

$t_{\text {heat }}=\frac{\rho_{\text {bulk }} c_{\mathrm{d}} s^{2}}{k_{\mathrm{th}}}$

where $c_{\mathrm{d}} \approx 1000 \mathrm{~W} \mathrm{~m}^{-1} \mathrm{~K}^{-1}$ is the particle's heat capacity.

We can use two different approaches to deal with rotation. One is to compare collision time to stopping time: if $t_{\text {coll }} / t_{\mathrm{s}} \gg$ 1, damping of rotation occurs faster than excitation by impacts, and therefore photophoresis is not significantly weakened. Using Eqs. (41) and (19), we get

$\frac{t_{\text {coll }}}{t_{\mathrm{s}}}=25 \frac{v_{\text {th }}}{v}$.

Another option is to check whether rotation, once it has been excited by collisions, is sufficiently slow to allow a stable heat gradient to be established within the particle: this is the case if $t_{\text {rot }} / t_{\text {heat }} \gg 1$. In order to find a rough estimate for $t_{\text {rot }}$, we assume that the particle transforms the entire kinetic energy of its radial motion $v_{\mathrm{r}}$ into rotational energy:

$\frac{1}{2}\left(\frac{2 \pi}{t_{\text {rot }}}\right)^{2} J_{\text {sphere }}=\frac{1}{2} m v_{\mathrm{r}}^{2}$,

with the solid sphere's moment of inertia $J_{\text {sphere }}=(2 / 5) m s^{2}$, we get

$t_{\text {rot }}=\sqrt{\frac{8}{5}} \frac{\pi s}{v_{\mathrm{r}}}$.

Figure 10 shows $t_{\text {coll }} / t_{\mathrm{s}}$ as a function of $v$ and $t_{\text {rot }} / t_{\text {heat }}$ as a function of $s$. The distance is chosen to be $r=10 \mathrm{AU}$. We see that collisional timescales are longer than stopping timescales by many decades, thus allowing rotation to decline between collisions. Nonetheless, rotation frequencies can be high enough to cancel out stable temperature gradients. We conclude that photophoresis can be suppressed directly after collisions through rapid rotation, but remains effective for most of the time because gas drag damps spin faster than collisions can excite it. 

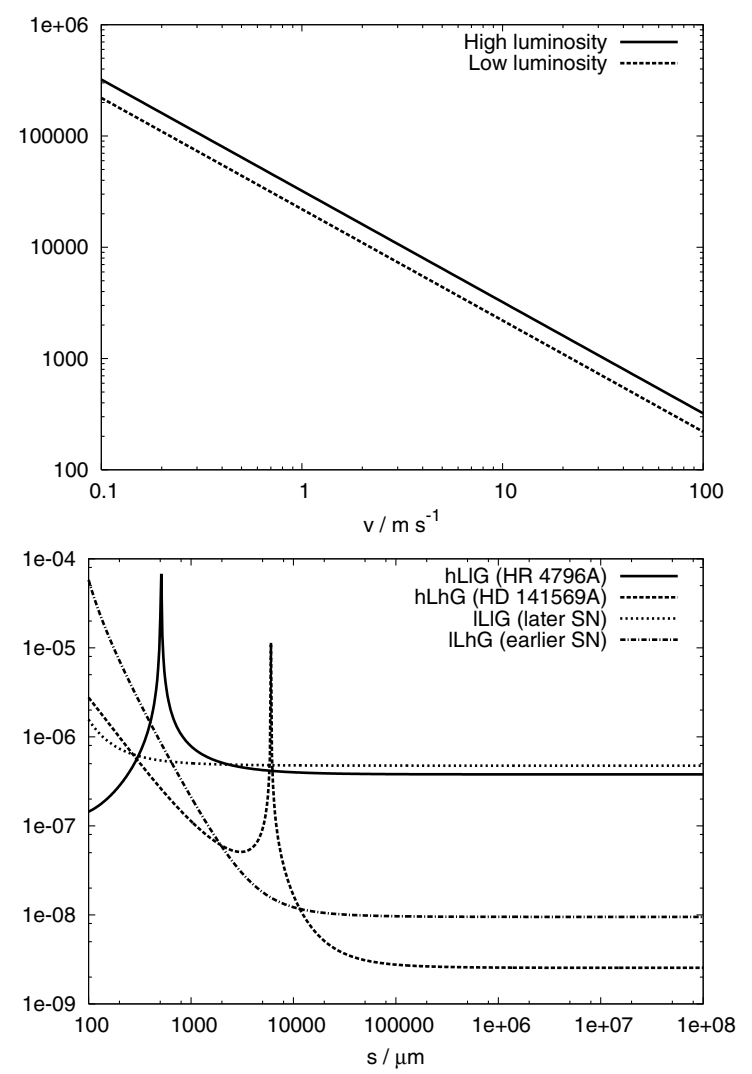

Fig. 10. Ratios of typical timescales. Top: $t_{\text {coll }} / t_{\mathrm{s}}$ as a function of $v$. Bottom: $t_{\text {rot }} / t_{\text {heat }}$ as a function of $s$.

\section{Conclusions and discussion}

In this paper, we have analyzed the effect of photophoresis on the dynamics of solid particles in the optically-thin, yet sufficiently gas-rich, transitional disks around young stars. To this end, we employed a single-body approach and added the photophoretic force to the standard array of perturbing forces (stellar gravity, direct radiation pressure, and gas drag). Particle-particle interactions as well as particle growth were not included. We find that photophoresis may lead to noticeable corrections to the results obtained with models that do not take it into account.

Our main results can be summarized as follows:

1. Both with and without photophoresis, solid objects migrate inward or outward, until they reach the stability distance $r_{\text {stab }}(s)$, where $s$ is the particle radius. At that distance, radial forces cancel each other in the particle's own inertial system, and the orbital velocity is equal to that of the gas. The stability distance is a decreasing function of $s$, therefore particles are sorted according to size, with larger bodies accumulating closer to the star. These results fully agree with those by Takeuchi \& Artymowicz (2001).

2. Photophoresis increases the stability radii, moving objects to larger radial distances. The effect is noticeable in the size range from several micrometers to several centimeters (for older transitional disks) or even several meters (for younger, more gaseous, ones).

3. The steady-state distribution of solids is completely characterized by the function $r_{\text {stab }}(s)$, the shape of which depends on the system's parameters:

- Higher gas densities move the curve to larger distances without changing its overall shape. Gas density also controls the maximum particle size up to which stable orbits can exist.

- The steepness of the gas density radial profile determines the slope of $r_{\mathrm{stab}}(s)$, with flatter profiles generating steeper curves.

- Stellar luminosity determines the curve's shape. While high luminosities $\left(\sim 20 L_{\odot}\right)$ produce simple decreasing curves, low (solar) luminosities generate a plateau at a distance from the star that can be computed with the aid of Eq. (35). In this area, objects in a certain size range may accumulate.

4. Particle rotation tends to reduce the photophoretic effect. Our estimates suggest, however, that it is damped by gas drag quickly enough to keep photophoresis at work.

Our work predicts the formation of a particle concentration belt at a certain distance from the star. For the high luminosity systems, it is not very pronounced (hLhG) or does not appear at all (hL1G). Furthermore, the radii of the observed rings around HD 141569A are an order of magnitude larger than that of the slight concentration belt predicted for the hLhG system. It is not likely therefore that the observed structures around HR 4796A and HD 141569A are caused by photophoresis. While photophoresis is probably active in transitional disks, the circumstellar rings of HR 4796A and HD 141569 must be shaped by other forces and effects, such as gravitational sculpting by planets or interactions with stellar companions. Alternatively, a rapid decline of gas density at the disks' edges (Takeuchi \& Artymowicz 2001) or a recently proposed dust-gas instability (Besla \& Wu 2007) may cause particles to accumulate there.

The model presented here is rather exploratory and rests on a number of simplifying assumptions. In the future, we plan to investigate the problem a more realistic way, lifting some of the assumptions we made to keep the problem tractable. First, we plan to deal with particle-particle interactions, taking collisions and growth into account. This can be done in the style of Krauss et al. (2006), increasing the radius through an exponential ansatz $s=s_{0} \exp \left(t / t_{0}\right)$. The latter corresponds to the assumption that the object moving through the nebula collects smaller particles on its surface. A more detailed approach will employ statistical methods (Krivov et al. 2005, 2006). Then, we wish to explore rotation in greater detail, calculating collision timescales and rotation frequencies using models for two-body collisions. Also, the effects of gas drag and radiation forces on non-spherical objects need to be taken into account (see e.g. Xu et al. 1999). Other issues include the variation of physical parameters (density, thermal conductivity, emissivity, $J_{1}$ ) with size and distance from the star, as well as the global evolution and clearing of dust in the system which defines the time around which photophoresis can come into play.

In spite of these unknowns, we have demonstrated that photophoretic force in transitional circumstellar disks cannot be neglected, and has to be included in elaborate models of such systems.

Acknowledgements. We wish to thank Gerhard Wurm for useful discussions, Jürgen Blum and Bo Gustafson for pointing out several papers on the photophoretic effect and the anonymous referee for helpful comments. F.H. is supported by the graduate student fellowship of the Thuringia State.

\section{References}

Alibert, Y., Mordasini, C., Benz, W., \& Winisdoerffer, C. 2005, A\&A, 434, 343 Ardila, D. R., Lubow, S. H., Golimowski, D. A., et al. 2005, ApJ, 627, 986 Arnold, S., \& Lewittes, M. 1982, J. Appl. Phys., 53, 5314 
Augereau, J.-C. 2006, in Semaine de l'Astrophysique Française, ed. D. Barret, F. Casoli, G. Lagache, A. Lecavelier, \& L. Pagani, 375

Augereau, J. C., \& Papaloizou, J. C. B. 2004, A\&A, 414, 1153

Augereau, J. C., Lagrange, A. M., Mouillet, D., \& Ménard, F. 1999a, A\&A, 350, L51

Augereau, J.-C., Lagrange, A.-M., Mouillet, D., Papaloizou, J. C. B., \& Grorod, P. A. 1999b, A\&A, 348, 557

Balog, Z., Muzerolle, J., Rieke, G. H., et al. 2007, ApJ, 660, 1532

Beresnev, S., Chernyak, V., \& Fomyagin, G. 1993, Phys. Fluids A, 5, 2043

Besla, G., \& Wu, Y. 2007, ApJ, 665, 528

Blum, J., \& Wurm, G. 2000, Icarus, 143, 138

Bouwman, J., Meyer, M. R., Kim, J. S., et al. 2006, in Planet formation: theory, observations and experiments, ed. H. Klahr, \& W. Brandner, 14

Brauer, F., Dullemond, C. P., Johansen, A., et al. 2007, A\&A, 704, 1169

Burns, J. A., Lamy, P. L., \& Soter, S. 1979, Icarus, 40, 1

Calvet, N., D’Alessio, P., Watson, D. M., et al. 2005, ApJ, 630, L185

Chen, C. H., \& Kamp, I. 2004, ApJ, 602, 985

Currie, T., Balog, Z., Kenyon, S. J., et al. 2007, ApJ, 659, 599

Draine, B. T., \& Weingartner, J. C. 1996, ApJ, 470, 551

Ehrenhaft, F. 1917, Phys. Z., 18, 352

Eisner, J. A., Chiang, E. I., \& Hillenbrand, L. A. 2006, ApJ, 637, L133

Fisher, R. S., Telesco, C. M., Piña, R. K., Knacke, R. F., \& Wyatt, M. C. 2000, ApJ, 532, L141

Haisch, Jr., K. E., Jayawardhana, R., \& Alves, J. 2005, ApJ, 627, L57

Hayashi, C., Nakazawa, K., \& Nakagawa, Y. 1985, in Protostars and Planets II, ed. D. C. Black, \& M. S. Matthews, 1100

Henning, T., Dullemond, C. P., Wolf, S., \& Dominik, C. 2006, in Planet formation: Theory, observations and experiments, ed. H. Klahr, \& W. Brandner, 112

Hollenbach, D., Johnstone, D., Lizano, S., \& Shu, F. 1994, ApJ, 428, 654

Hollenbach, D., Gorti, U., Meyer, M., et al. 2005, ApJ, 631, 1180

Hollenbach, D. J., Yorke, H. W., \& Johnstone, D. 2000, in Protostars and Planets IV, ed. V. Mannings, A. Boss, \& S. Russell (University of Arizona Press), 401

Hueso, R., \& Guillot, T. 2005, A\&A, 442, 703

Jayawardhana, R., Fisher, S., Hartmann, L., et al. 1998, ApJ, 503, L79

Jayawardhana, R., Coffey, J., Scholz, A., Brandeker, A., \& van Kerkwijk, M. H. 2006, ApJ, 648, 1206

Jura, M., Zuckerman, B., Becklin, E. E., \& Smith, R. C. 1993, ApJ, 418, L37

Jura, M., Malkan, M., White, R., et al. 1998, ApJ, 505, 897

Kastner, J. H., Huenemoerder, D. P., Schulz, N. S., et al. 2001, in Young Stars Near Earth: Progress and Prospects, ed. R. Jayawardhana, \& T. Greene, ASP Conf. Ser., 244, 159

Klahr, H. H., \& Lin, D. N. C. 2000, in Disks, Planetesimals and Planets, ed. F. Garzón, C. Eiroa, D. de Winter, \& T. J. Mahoney, ASP Conf. Ser., 219, 375

Kley, W., Papaloizou, J. C. B., \& Lin, D. N. C. 1993, ApJ, 416, 679

Koerner, D. W., Ressler, M. E., Werner, M. W., \& Backman, D. E. 1998, ApJ, 503, L83

Krauss, O., \& Wurm, G. 2005, ApJ, 630, 1088

Krauss, O., Wurm, G., Moussis, O., et al. 2006, A\&A, 462, 997

Krivov, A. V., Sremčević, M., \& Spahn, F. 2005, Icarus, 174, 105

Krivov, A. V., Löhne, T., \& Sremčević, M. 2006, A\&A, 455, 509

Lawson, W. A., Lyo, A.-R., \& Muzerolle, J. 2004, MNRAS, 351, L39

Lissauer, J. J. 1993, ARA\&A, 31, 129

Lowrance, P. J., Schneider, G., Kirkpatrick, J. D., et al. 2000, ApJ, 541, 390
Mackowski, D. W. 1989, Int J. Heat Mass Transfer, 32, 843

Meyer, M. R., Backman, D. E., Weinberger, A. J., \& Wyatt, M. C. 2007, in Protostars and Planets V, ed. B. Reipurth, D. Jewitt, \& K. Keil, 573

Meyer, M. R., \& Beckwith, S. V. W. 2000, in Lecture Notes in Physics (Berlin: Springer Verlag) ISO Survey of a Dusty Universe, ed. D. Lemke, M. Stickel, $\&$ K. Wilke, 548, 341

Morfill, G. E. 1983, Icarus, 53, 41

Morfill, G. E. 1988, Icarus, 75, 371

Mouillet, D., Lagrange, A. M., Augereau, J. C., \& Ménard, F. 2001, A\&A, 372, L61

Paddack, S. J., \& Rhee, J. W. 1975, Geophys. Res. Lett., 2, 365

Papaloizou, J. C. B., \& Terquem, C. 1999, ApJ, 521, 823

Papaloizou, J. C. B., Terquem, C., \& Nelson, R. P. 1999, in Astrophysical Discs - an EC Summer School, ed. A. J. Sellwood, \& J. Goodman, ASP Conf. Ser., 160,186

Rohatschek, H. 1996, J. of Aerosol Sc., 27, 467

Safronov, V. S. 1972, Evolution of the Protoplanetary Cloud and Formation of the Earth and Planets, NASA TTF-677

Shakura, N. I., \& Sunyaev, R. A. 1973, A\&A, 24, 337

Shu, F. H., Adams, F. C., \& Lizano, S. 1987, ARA\&A, 25, 23

Simon, M., \& Prato, L. 1995, ApJ, 450, 824

Stauffer, J. R., Hartmann, L. W., \& Barrado y Navascues, D. 1995, ApJ, 454, 910

Steinbach, J., Blum, J., \& Krause, M. 2004, Europ. Ph. J. E, 15, 287

Strom, S. E., \& Edwards, S. 1993, in Planets Around Pulsars, ed. J. A. Phillips,

S. E. Thorsett, \& S. R. Kulkarni, ASP Conf. Ser., 36, 235

Strom, S. E., Edwards, S., \& Skrutskie, M. F. 1993, in Protostars and Planets III, ed. E. H. Levy, \& J. I. Lunine, 837

Takeuchi, T., \& Artymowicz, P. 2001, ApJ, 557, 990

Takeuchi, T., Clarke, C. J., \& Lin, D. N. C. 2005, ApJ, 627, 286

Tehranian, S., Giovane, F., Blum, J., Xu, Y.-L., \& Gustafson, B. A. S. 2001, Int. J. Heat Mass Transfer, 44, 1649

Telesco, C. M., Fisher, R. S., Piña, R. K., et al. 2000, ApJ, 530, 329

Thébault, P., \& Augereau, J.-C. 2005, A\&A, 437, 141

van den Ancker, M. E., de Winter, D., \& Tjin A Djie, H. R. E. 1998, A\&A, 330, 145

Wahhaj, Z., Koerner, D. W., Backman, D. E., et al. 2005, ApJ, 618, 385

Weidenschilling, S. J. 1977, MNRAS, 180, 57

Weidenschilling, S. J., \& Cuzzi, J. N. 1993, in Protostars and Planets III, ed. E. H. Levy, \& J. I. Lunine, 1031

Weinberger, A. J., Becklin, E. E., Schneider, G., et al. 1999, ApJ, 525, L53

Weinberger, A. J., Rich, R. M., Becklin, E. E., Zuckerman, B., \& Matthews, K. 2000, ApJ, 544, 937

Wetherill, G. W. 1980, ARA\&A, 18, 77

Wieneke, B., \& Clayton, D. D. 1983, in Chondrules and their Origins, ed. E. A. King, 284

Wurm, G., \& Blum, J. 2006, in Planet formation: Theory, observations and experiments, ed. H. Klahr, \& W. Brandner, 90

Wurm, G., \& Krauss, O. 2006, Icarus, 180, 487

Wyatt, M. C. 2005, A\&A, 440, 937

Wyatt, M. C., Dermott, S. F., Telesco, C. M., et al. 1999, ApJ, 527, 918

Xu, Y.-L., Gustafson, B. A. S., Giovane, F., Blum, J., \& Tehranian, S. 1999, Phys. Rev. E, 60, 2347

Zuckerman, B., \& Becklin, E. E. 1993, ApJ, 406, L25

Zuckerman, B., \& Song, I. 2004, ARA\&A, 42, 685 